\title{
Soil Improvement of EPBS Construction in High Water Pressure and High Permeability Sand Stratum
}

\author{
Shimin Wang, ${ }^{1}$ Xixi Lu, ${ }^{1}$ Xianming Wang, ${ }^{1}$ Chuan He ${ }^{D},{ }^{1}$ Xin Xia, ${ }^{2}$ Lei Ruan, \\ and Yunqi Jian ${ }^{1}$ \\ ${ }^{1}$ Key Laboratory of Transportation Tunnel Engineering, Ministry of Education, Southwest Jiaotong University, \\ Chengdu 610031, China \\ ${ }^{2}$ China Railway Second Academy Subway Institute, Chengdu 610031, China
}

Correspondence should be addressed to Chuan He; chuanhe21@swjtu.edu.cn

Received 24 November 2018; Accepted 23 February 2019; Published 29 May 2019

Academic Editor: Castorina S. Vieira

Copyright (C) 2019 Shimin Wang et al. This is an open access article distributed under the Creative Commons Attribution License, which permits unrestricted use, distribution, and reproduction in any medium, provided the original work is properly cited.

A mud-water balance shield is the preferred method for the high permeability and high water pressure stratum, but when restricted by construction and environmental conditions, the shield must be constructed with the earth pressure balance shield (EPBS). The risk of slag spraying is extremely high during construction, and this often results in great hidden dangers that threaten workers' safety during the construction of the tunnel. Slag improvement is an effective measure to prevent slag spraying, and the relevant research is bringing more and more attention to this activity. Based on the background for the tunnel project of Shangteng-Dadao Section of the No. 1 line for Fuzhou Rail Transit, ratio method is proposed for the combined application of sodium bentonite-carboxymethyl cellulose-polyacrylamide adopted by laboratory test focusing on the challenge of residual soil spraying for earth pressure balance shield under the stratum with high water pressure and high permeability; consequently, the best improvement program is suggested for the construction using earth pressure balance shield on this typical kind of stratum, which can be briefly described as the respective mixing of $2.5 \%$ and $0.5 \%$ accounting for the sand volume by swelling soil with a concentration of $10 \%$, sodium bentonite-CMC mixed mud with a concentration of $5 \%$, and polyacrylamide solution with a concentration ranging from $3 \%$ to $4 \%$; furthermore, the results for the field application are random inspected.

\section{Introduction}

Since the first use of EPBS in 1974, with the continuous development of technology, it has been applied in more than $90 \%$ of the shield tunnel projects in the world [1]. Due to its small impact on the surrounding environment, strong adaptability to complex geological conditions, and safe and rapid construction, EPBS gradually has become the preferred method for constructing tunnels under rivers or the sea. However, when the shield machine is burrowing in a stratum with high permeability, such as gravel stratum, the stratum often exhibits large gaps between the particles. When this occurs, the lack of a cohesive force and poor plasticity of the soil often result in the slag in the soil tank being prone to various problems, such as poor gradation, strong water permeability, and poor workability. In an environment in which the water pressure is high, the groundwater in the shield earth chamber can easily penetrate the slag and seep out along the shield machine hopper, which creates risks during normal construction and easily causes slag spraying. Thus, the construction safety of the shield tunnel is seriously threatened. The schematic diagram of EPBS slag spraying is shown in Figure 1.

Scholars from different countries have conducted various theoretical and experimental studies on the EPBS spraying. Some scholars have conducted related research on the mechanism of slag spraying [2-8], which has provided a certain guiding role for the prevention and control of slag spraying. At the same time, the equipment will be improved during the construction process to reduce the risk of slag spraying $[9,10]$. However, in many solutions, turning the sandy soil improvement into impervious cohesive soil is the most intuitive and effective method to solve slag spraying [11]. Many scholars have conducted research on soil 


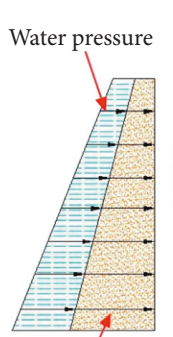

Earth pressure

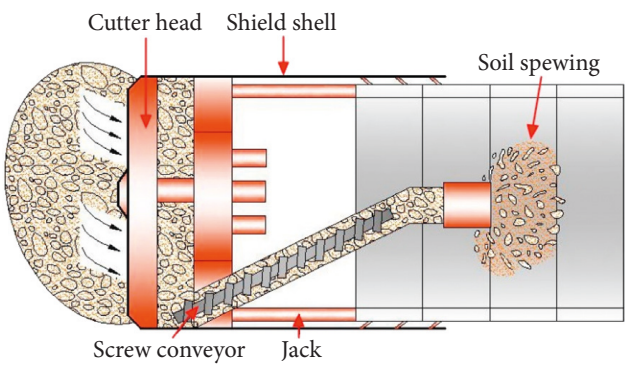

FIGURE 1: Schematic diagram of EPBS slag spraying. improvement for different stratum conditions and water pressure conditions [12-14].

Currently, there are many kinds of materials that can be added to improve the soil, such as representative interface materials and mineral modifiers, foaming agents and bentonites, which are used in most projects due to their relatively low cost and extensive applicability. Researchers around the world also have continued to study how to improve the soil. Quebaud et al. [15], Sotiris [16], and Xavier [17] have discussed the importance of foam-improved soils for EPBS construction. Laboratory experiments of Peila et al. $[18,19]$ show that the use of foaming agent for soil improvement can enhance the adaptability of EPBS in a rock stratum. Ehsan et al. [20] found that the use of foam for soil improvement can improve the flow plasticity of the slag, thereby reducing the internal friction of the soil and the torque during EPBS tunneling. Some scholars have conducted research on the microscopic properties and the puffing mechanism of mineral modifiers represented by bentonite. He et al. [21] discussed the optimal concentration and optimal volume ratio of the improved sand-cobble stratum with sodium bentonite, and they proved that the improved slag has high spraying control reliability. The results of Martinelli et al. [22] showed that the combination of a foaming agent and bentonite can achieve a better soil improvement effect when EPBS is used in the oil sand layer.

The existing research indicates that the soil improvement of EPBS is focused mainly on the performance of a single material, but there is still less research on the combination and performance of different improved materials under high water pressure and high permeability. The permeability coefficient of the permeable stratum involved in the current research basically is no more than $10^{-2} \mathrm{~cm} / \mathrm{s}$, and the water pressure to which the shield is subjected during construction usually is not high. However, with the extensive application of shield technology, some stratum conditions with high permeability and water pressure must be constructed by EPBS due to the limitations of the construction environment. The risk of slag spraying during construction is extremely high, which is a huge threat to the safety of the people involved. Therefore, in this paper, our intent was to use laboratory tests to study the soil improvement scheme of the EPBS under high water pressure and high permeability relying on the tunnel of the Shangteng-Dadao Section on the No. 1 line of Fuzhou Rail Transit. The tests were conducted by selecting various test materials and comprehensively considering the permeability and workability of the slag.
Subsequently, the laboratory test results were applied in an actual project to test their effect in the project.

\section{Project Overview}

The total length of the tunnel at the Shangteng-Dadao station on the No. 1 line of the Fuzhou Rail Transit is about $1700 \mathrm{~m}$. The strata in the river section are mainly fine sand, medium sand, silt soil with a thin silt layer, and a small amount of sand-clay. The distribution of the geological conditions is shown in Figure 2. The tunnel passes mainly through the middle sand stratum, which is $30-35 \mathrm{~m}$ deep. According to the geochemical survey, the maximum permeability coefficient of the middle sand layer can reach $10^{-2} \mathrm{~cm} / \mathrm{s}$. The stratum is located in the Minjiang River section, and the peak water head in the upper part reaches $40 \mathrm{~m}$ due to the influence of tides, so the shield crosses the stratum as a typical high pressure and high permeability sandy stratum. Due to the construction environment and the construction scheme of the adjacent section, the tunnel at Shangteng-Dadao Station was constructed by the EPBS tunneling under the Minjiang River. During construction, the risk of slag spraying is extremely high.

To prevent spraying in the supersaturated sandy stratum, the key point is to reduce the permeability coefficient of the slag and control its viscosity and flow plasticity quantified by the slump cone test [23-29]. According to the experience that has been gained in constructing sand shields in Shenzhen, Guangzhou, Nanjing, and other locations, it is evident that shield spraying accidents can be completely suppressed when the soil permeability coefficient has been improved and is in the range of $(1-2) \times 10^{-5} \mathrm{~cm} / \mathrm{s}$ [30]. Many scholars, such as Vinai et al. [31], Anagnostou and Kovari [32], also found that almost no water permeability occurred when the improved slag permeability coefficient was decreased to $1 \times 10^{-5} \mathrm{~cm} / \mathrm{s}$. Therefore, the target value of the improved slag permeability coefficient was considered to be less than $1 \times 10^{-5} \mathrm{~cm} / \mathrm{s}$.

For viscosity of soil, by referring to the technical indicators for the construction of the existing metro EPBS machine, its target determined was less than $100 \mathrm{~s}$. And for the improvement of soil collapsed slump, Quebaud et al. [15] stated that the plastic flow state for residual soil after improvement is most suitable for requirements of the shield machine pressure regulation and conveying when the collapsed slump becomes $12 \mathrm{~cm}$; Vinai et al. [31] posited that the plastic flow state of the improved slag is the most suitable when the slump is $120-185 \mathrm{~mm}$. Extensive experience with EPBSs in China has shown that the soil slump can meet the requirement of the plastic flow state when the slump is in the range of $10-15 \mathrm{~cm}$. Therefore, the target value of slump, as determined in comprehensive laboratory tests, is $10-15 \mathrm{~cm}$.

\section{Soil Improvement Test of the Supersaturated Sandy Stratum}

For high permeability and high water pressure stratum, during the construction process of shield tunneling, the shield machine is commonly in the state of full warehouse during excavation in case that excessive groundwater fills in 


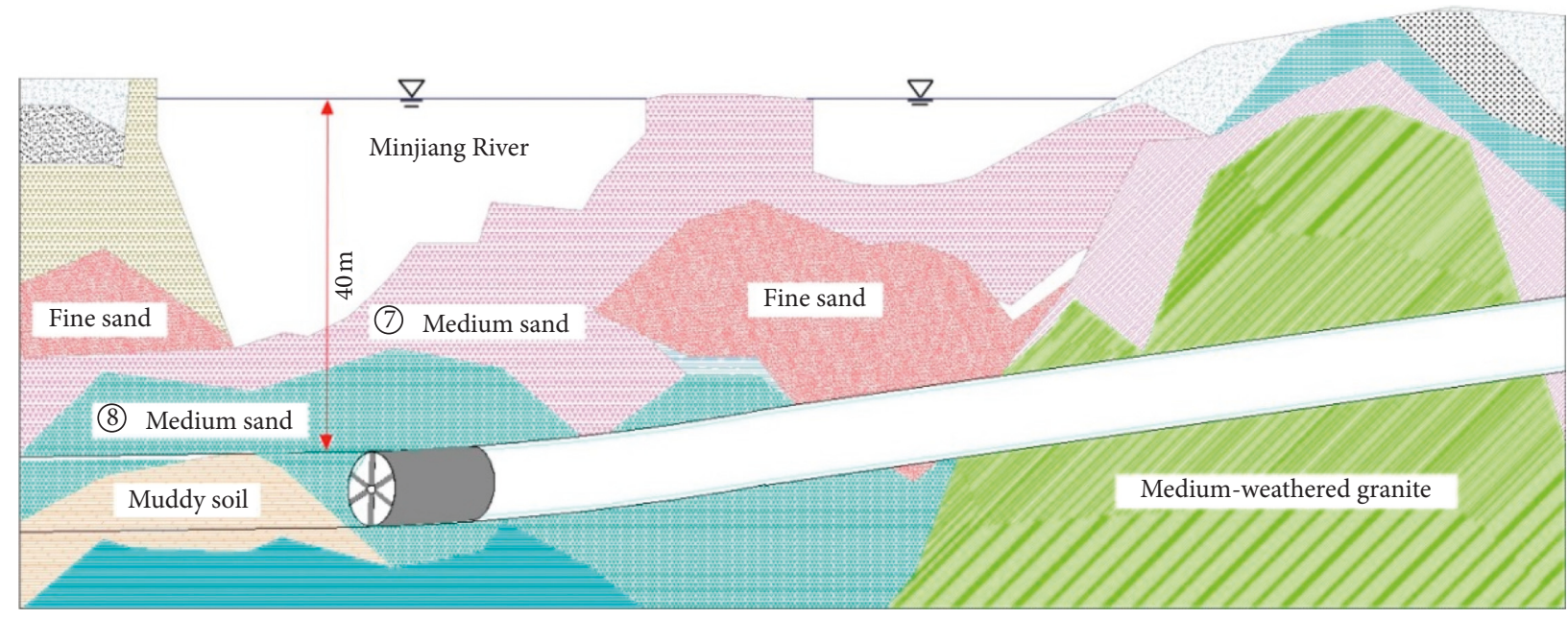

FIGURE 2: Geological diagram of the section under the river.

the space of soil warehouse and potentially increases the risk of slag spraying. At this time, the tank muck is a mixture of water and sand, and the sand is compacted and supersaturated with the water filling the spaces between the particles of sand. Therefore, supersaturated sand is used as a soil improvement material in the laboratory test.

\subsection{Preparation of Soil Sample of Supersaturated Sandy} Stratum. The slag of the adjacent section shield tunneling was sampled and sieved, and the medium sand particle gradation is shown in Table 1. According to the gradation, the river sand is self-purchased, dried, and sieved, and then, the medium sand will be fully reshaped. The coefficient of the permeability for the remolded medium sand is determined by the constant head penetration test, and then, the sand will be filled to the supersaturated state by feeding water. The process of producing a sample of the sand is shown in Figure 3.

The control of the amount of water is very important when adding water to make a remolding sand sample supersaturated. Therefore, when mixing saturated sand in the laboratory, first a certain amount of dry sand is poured into the bucket, and the surface is leveled. Then, water is added slowly until the surface of the water just spills over the surface of the sand. Figure 4 shows a sample of supersaturated sand.

\subsection{Improvement Effect of Sodium Bentonite-CMC Mixed} $M u d$. At present, foam is commonly used as the modifier for residual soil of the shield tunnel in China; however, the pores for saturated sandy stratum are relatively large, and bubbles cannot be easily filled in sand grains; as a matter of fact, the permeability coefficient cannot be reduced under the circumstances, considering that the volume of bentonite will be expanded encountering water, resulting in the volume increase for particles; impermeability can be improved by the modifier filled in the gaps of residual soil; in consequence, the improvement on the gradation of residual soil can be conducted by sodium bentonite [33]. Xia [34] used sodium bentonite for the improvement of the soil, which shows that the viscosity of the mud is positively correlated with the concentration of bentonite. When the concentration of bentonite is $1: 8$, the viscosity of the mud reaches $90 \mathrm{~s}$, meeting the viscosity standard; when the ratio of bentonite is $10: 2 \sim 10: 2.5$, the slump of bentonite meets the standard from 10 to $15 \mathrm{~cm}$; however, when the viscosity and slump standards are met at the same time, the evaluation criteria of the permeability coefficient less than $1 \times 10^{-5} \mathrm{~cm} / \mathrm{s}$ cannot be satisfied. Considering that CMC has a sticky effect on soil particles, it can play the role of retaining water, increasing the consistency, and reducing the permeability coefficient of the muck [35]; therefore, the sodium bentonite-CMC mixed mud is used for the improvement of the slag, but CMC solution is $2 \%$, and its viscosity has exceeded the range of shield pumping capacity [34], and so, it is only used as an auxiliary material. [36-38].

Based on the existing research, two different sodiumbased bentonite slurry with viscosity close to the upper limit of $1: 10$ and $1: 8$ are used as the base modifiers to configure the sodium-based bentonite-CMC mixed mud of two schemes. The CMC concentrations were, respectively, $1 \%$, $2.5 \%$, $5 \%$, $7.5 \%$, and $10 \%$. Details of the scheme are shown in Table 2.

The test sand was mixed with the sodium bentoniteCMC mixed slurry of the above various schemes according to the solid-liquid doping ratio of $10: 2$, and the sand permeability coefficient of each scheme was measured. The results are shown in Table 3.

The antipermeability of the supersaturated sand obviously was improved when the sodium bentonite solution is added with CMC, as shown in Table 3, and the improved permeability coefficient of the sand was less than the limit value of $1 \times 10^{-5} \mathrm{~cm} / \mathrm{s}$ of the different $\mathrm{CMC}$ concentrations. The results show that the sodium bentonite-CMC mixed mud can improve the impermeability of the slag. At the same time, it can be seen that from schemes 1-1 to 1-5 and schemes 2-1 to 2-5, with the CMC concentration gradually increasing, the rate of decrease of the permeability coefficient gradually slows down, and the outcome of improvement also tends to be approaching the limit. Therefore, based on scheme 1-5, the experimental variables were further divided. 
TABLE 1: Grading sand particles.

\begin{tabular}{lccccccc}
\hline Particle size $(\mathrm{mm})$ & $>20$ & $20-2$ & $2-0.5$ & $0.5-0.25$ & $0.25-0.075$ & $0.075-0.005$ & $<0.005$ \\
Percentage & 0 & 1.9 & 15.9 & 37.9 & 21.7 & 5.3 \\
\hline
\end{tabular}
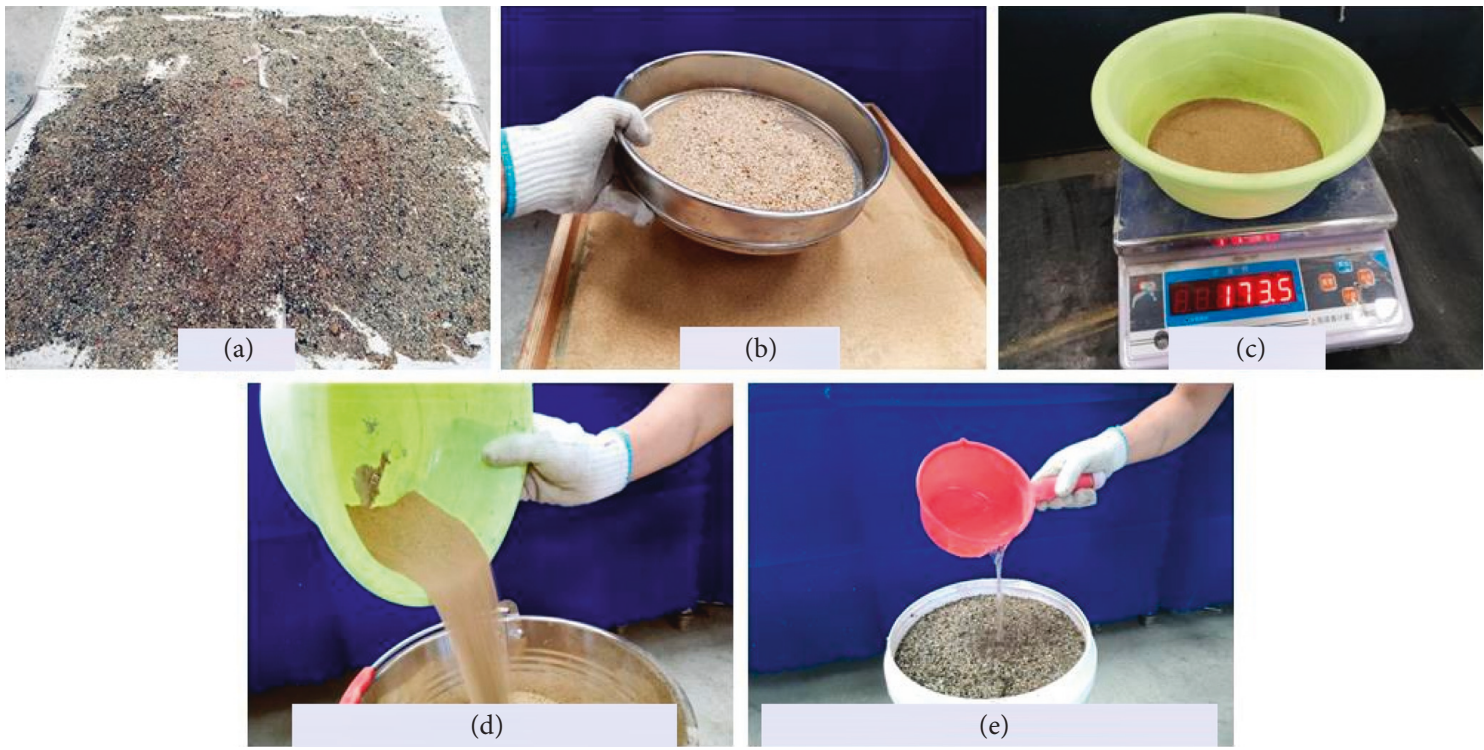

FIGURE 3: Remolding the sand configuration process.

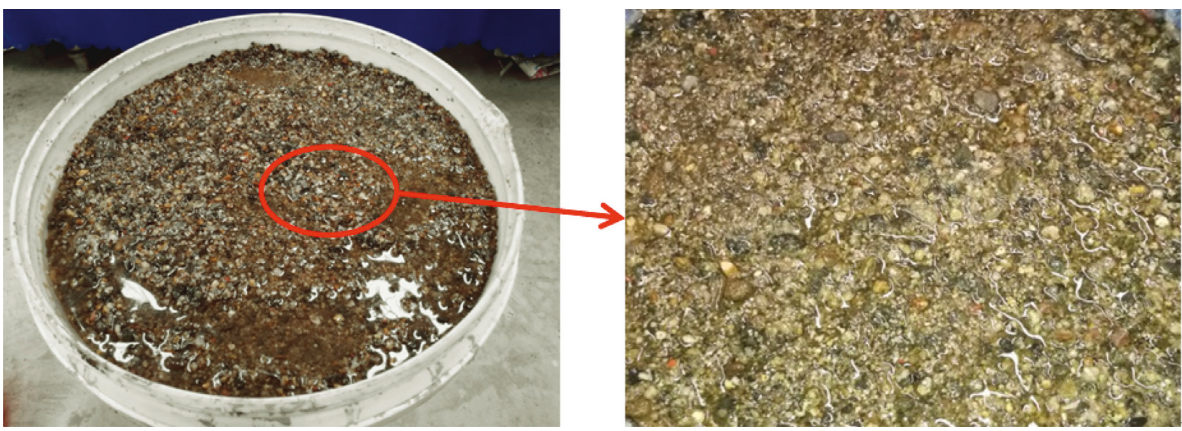

FIgURE 4: Remolding the supersaturation of medium sand.

TABLE 2: Sodium bentonite-CMC mixed mud ratio scheme.

\begin{tabular}{lccccc}
\hline Scheme 1 & CMC concentration (\%) & $\begin{array}{c}\text { Sodium bentonite-mixed } \\
\text { mud concentration }\end{array}$ & Scheme 2 & CMC concentration (\%o) & $\begin{array}{c}\text { Sodium bentonite-mixed } \\
\text { mud concentration }\end{array}$ \\
\hline $1-1$ & 1 & & $2-1$ & 1 & \\
$1-2$ & 2.5 & $1: 10$ & $2-2$ & 2.5 & $1: 8$ \\
$1-3$ & 5 & & $2-3$ & 5 & 7.5 \\
$1-4$ & 7.5 & & $2-4$ & 10 & \\
$1-5$ & 10 & & $2-5$ & & \\
\hline
\end{tabular}

TABLE 3: Permeability coefficients of supersaturated sand improved by sodium bentonite-CMC mixed mud.

\begin{tabular}{lrrrrrrrrrr}
\hline Scheme number & $1-1$ & $1-2$ & $1-3$ & $1-4$ & $1-5$ & $2-1$ & $2-2$ & $2-3$ & $2-4$ & $2-5$ \\
\hline $\begin{array}{l}\text { Permeability coefficients of supersaturated sand } \\
\left(\times 10^{-6} \mathrm{~cm} / \mathrm{s}\right)\end{array}$ & 7.31 & 4.72 & 1.75 & 0.769 & 0.265 & 6.47 & 2.96 & 0.961 & 0.415 & 0.138 \\
\hline
\end{tabular}

The concentrations of bentonite were, respectively, $8 \%, 9 \%$, $10 \%, 11 \%$, and $12 \%$, and the mass ratio of CMC to bentonite solution was, respectively, $2.5 \%$, $5 \%$, $7.5 \%$, and $10 \%$. The sodium-based bentonite-CMC mixed mud was prepared under the above conditions, and the corresponding viscosity test was carried out. The results are shown in Figure 5. 


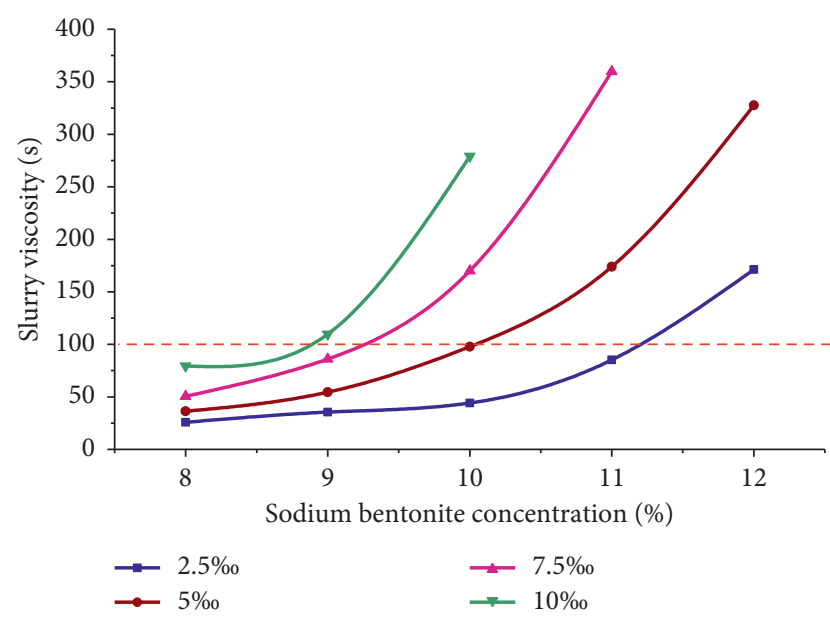

FIGURE 5: Viscosity curve of sodium-based bentonite-CMC mixed mud.

Figure 5 shows that the viscosity of the mixed mud increased remarkably when the amounts of bentonite and CMC were increased, and the viscosity of the partial mixing ratio mud exceeded the limit value of $100 \mathrm{~s}$. Therefore, the bentonite-CMC mixed mud that was close to the upper limit of the pumping capacity of the shield machine was selected for further experimental research. The selected sodium bentonite-CMC mixed mud parameters are shown in Table 4.

In order to study the effects of the mixing amount for sodium bentonite-CMC mixed mud on the permeability coefficient and slump of the supersaturated sand in the shielded soil tank after sieve, the supersaturated undisturbed sand is respectively mixed with sodium bentonite-CMC mixed mud whose number is, respectively, A, B, C, and $\mathrm{D}$ according to a volume ratio of $10: 1,10: 1.5,10: 2,10: 2.5$, and $10: 3$. The improved sand was subjected to a penetration test and a slump test, and the results of the tests are respectively shown in Figures 6 and 7.

When the mixing ratio of sodium bentonite-CMC mixed mud was increased, it was obvious that antipermeability of supersaturated sand was enhanced. When the mixing ratio of the mixed mud was less than $10: 2$, the improved coefficient of permeability of the sand was reduced. As the incorporation ratio continued to increase, the slag that was improved by the different proportions of mud attained a permeability coefficient less than $1 \times 10^{-5} \mathrm{~cm} / \mathrm{s}$, as shown as Figure $7(\mathrm{a})$. Compared with the single sodium bentonite solution, CMC will connect the fine particles in the mixed soil; thereby, the viscosity is increased for the soil near the excavation surface, and the permeability will be reduced for the soil; as a matter of fact, the improved slag will form a better waterproof sealing effect. From Figure 7(b), although the amount of mixed mud was increased, the supersaturated sand slump basically increased uniformly, and the slump loss was improved to a certain extent; however, the slump did not exceed $10 \mathrm{~cm}$ in the range of the solid-liquid volume ratios that were tested. Therefore, the sodium bentonite-CMC mixed mud solution cannot meet slump requirements in the supersaturated state.
3.3. Improved Effect of Sodium Bentonite-CMC Mixed Mud and Polyacrylamide Solution. Since the sodium-based bentonite-CMC mixed mud cannot be used to satisfy the requirements of the slump in the supersaturated state, it is considered to add an improver for improving the workability of the soil. Liu and Bai [30] showed that the slump is positively correlated with the polyacrylamide concentration and the blending ratio. The single-used polyacrylamide solution has a significant improving effect on the permeability and workability of the sand; therefore, on the basis of selecting the mix ratio and dosage of sodium bentonite-CMC mixed mud, a polyacrylamide solution was introduced, and the slag improvement test was carried out by adjusting the concentration and dosage of polyacrylamide.

In order to prevent the slump of the improved slag from being too large, refer to the study of Liu and Bai [30], taking the polyacrylamide concentration as $1 \%, 2 \%, 3 \%$, and $4 \%$, and the polyacrylamide blending ratio is $100: 3,100: 4,100$ : 5, and 100:6 for the cross test. For the mixing ratio and dosage of sodium bentonite-CMC mixed mud, considering the viscosity of the slurry itself and the effect of stopping the water on the slag, the numbered B sodium bentonite-CMC mixed mud was selected and is mixed with the supersaturated sand at $2.5: 10$. Figure 8 shows the improvement effect of polyacrylamide solution with different concentrations and blending ratios on supersaturated sand in the case of sodium bentonite-CMC mixed mud with the fixed mixing ratio and fixed blending ratio.

Figure 8(a) shows that the polyacrylamide incorporation enhanced the reduction of the improved permeability coefficient of the supersaturated sand, and the permeability of the slag met the requirements when the sodium-CMC mixed mud was mixed with the polyacrylamide solution. Figure 8(b) shows that the combination of the three improved materials made the supersaturated sand slump meet the requirements when the concentration of polyacrylamide was $3 \%$ and $4 \%$, and the volume ratio of incorporation was $100: 5$. Figure 9 shows the details.

By summarizing the properties of polyacrylamide, it was found that when the polyacrylamide solution was combined with the sodium bentonite-CMC mixed mud, the floc formed by the polyacrylamide solution enclosed the soil particles, the sodium bentonite particles, the CMC floc, and a large amount of water, all of which improved the workability of the saturated sand. The final improved scheme for supersaturated sand is shown in Table 5.

\section{Engineering Application}

After using the above formula to improve the slag at the construction site, the actual effect was evaluated comprehensively by observing the construction and using the sampling method to determine the slump and the coefficient of permeability. The improved slag at the construction site is shown in Figure 10. It was apparent that the improved supersaturated sand has a certain shape, and no obvious segregation phenomenon occurred. The slag is discharged continuously at the gate, and the large-grained sand can be 
TABLE 4: Sodium bentonite-CMC mixed mud mixing ratio grouping.

\begin{tabular}{lccc}
\hline Mixed mud proportioning number & Bentonite concentration (\%) & CMC quality ratio (\%) & Mud viscosity (s) \\
\hline A & 11 & 0.25 & 85.3 \\
B & 10 & 0.5 & 97.8 \\
C & 9 & 0.75 & 86 \\
D & 8 & 1 & 79.4 \\
\hline
\end{tabular}

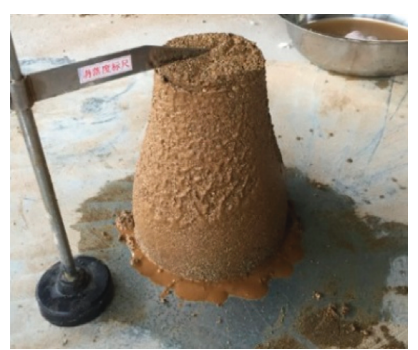

(a)

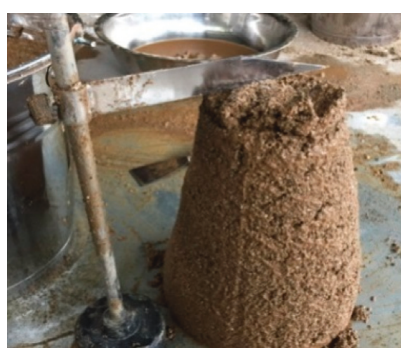

(b)

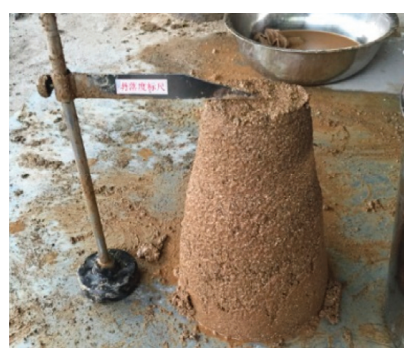

(c)

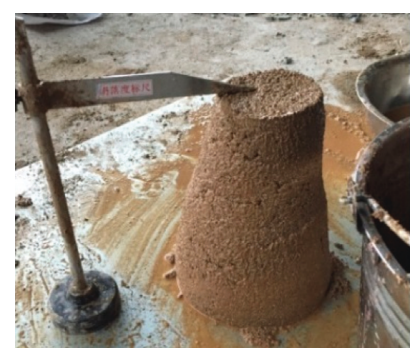

(d)

Figure 6: Slump of different matching when the solid-liquid volume ratio was 10:3. (a) Matching A. (b) Matching B. (c) Matching C. (d) Matching D.

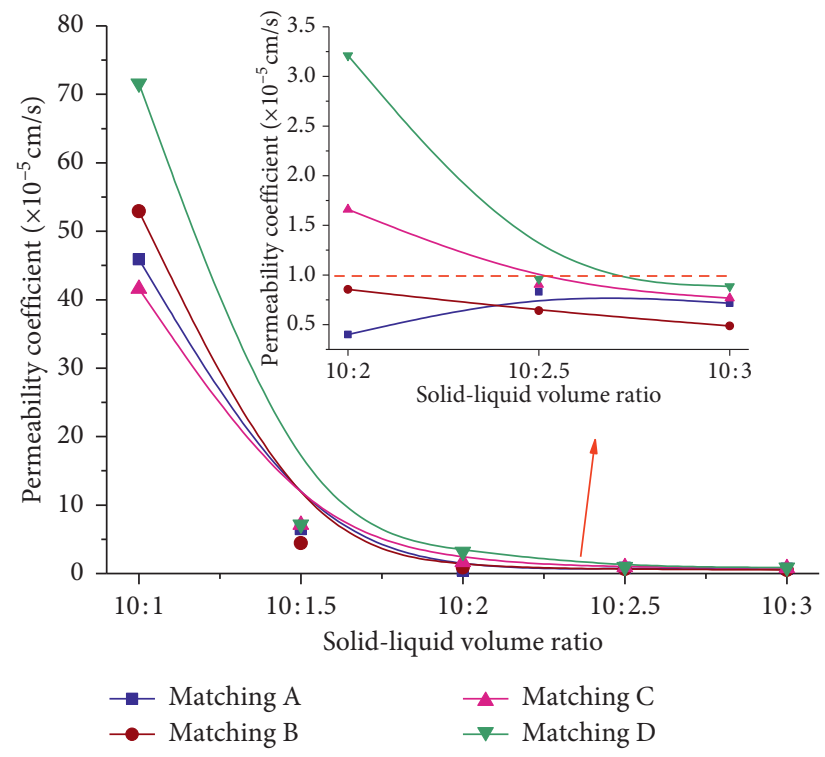

(a)

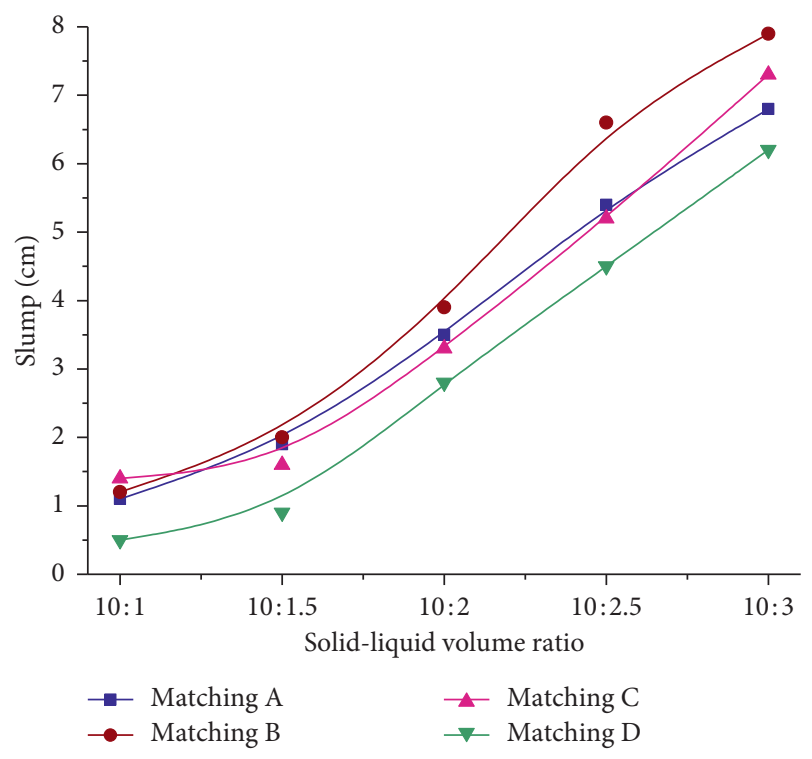

(b)

FIGURE 7: Improved variation in slag performance with the volume ratio. (a) Slag permeability coefficient. (b) Slag slump.

wrapped by the improved material because it has better flow plasticity.

At the same time, for the different excavation sections and the different operating conditions of the shield machine, the slag samples were taken randomly at the spiral excavator gate, and the slump and penetration tests were conducted. The results are shown in Table 6.

Table 6 shows that the improved slag performance index basically meets the target value, but the permeability coefficient of the slag generally was smaller than the laboratory test results, and the slump generally was too large. The main reason is that the soil in the shield soil tank can effectively control the water content in the soil tank after mixing with the improved material, so that the water content of the soil is significantly reduced. Therefore, it can be seen that the sodium-based bentonite-carboxymethyl cellulose-polyacrylamide ratio scheme can effectively solve the spraying problem of the earth pressure balance shield construction in high water pressure and high permeability sand formation.

\section{Conclusion}

In order to effectively avoid the occurrence of slag spraying in the construction of earth pressure balance shield in supersaturated sandy stratum, the laboratory soil improvement test was taken as a research method. Based on the 


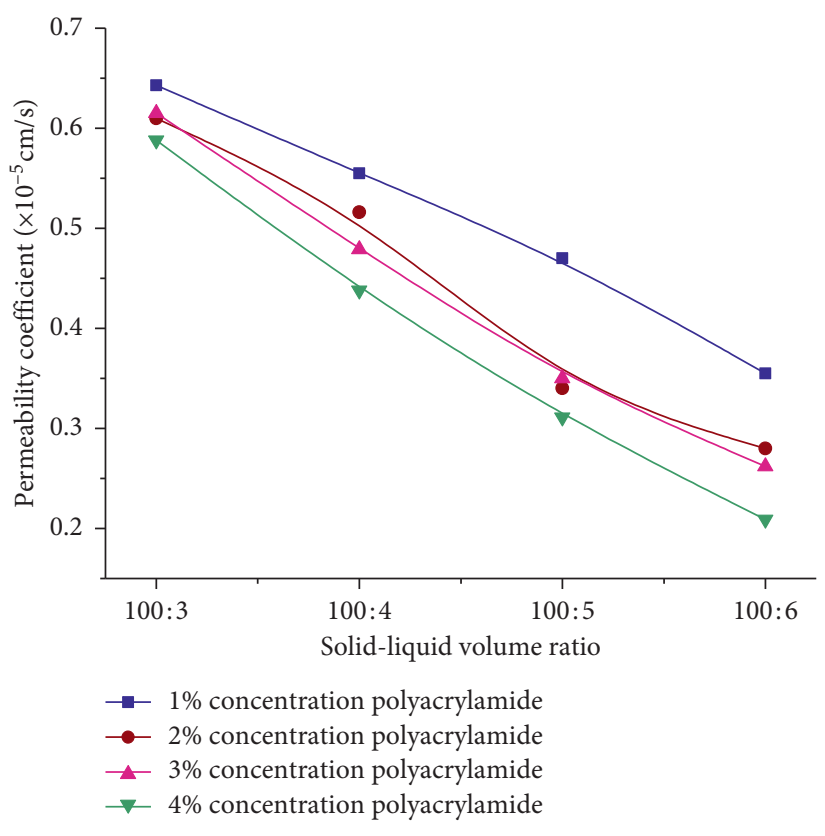

(a)

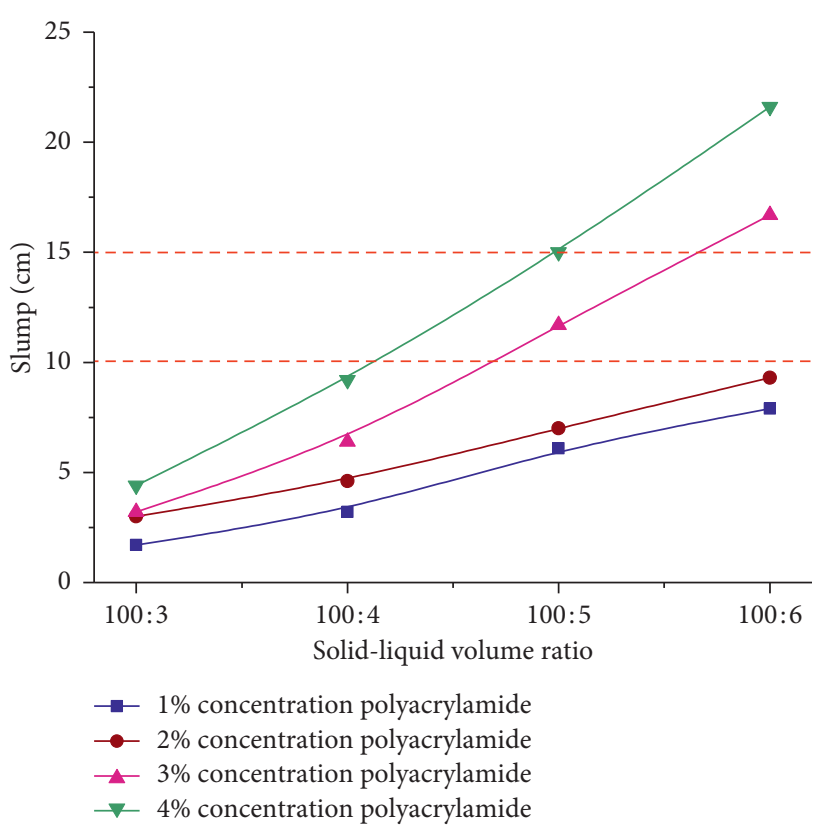

(b)

FIGURE 8: Improvement of the performance of the slag with the solid-liquid volume ratio of mixed mud. (a) Slag permeability coefficient. (b) Slag slump.

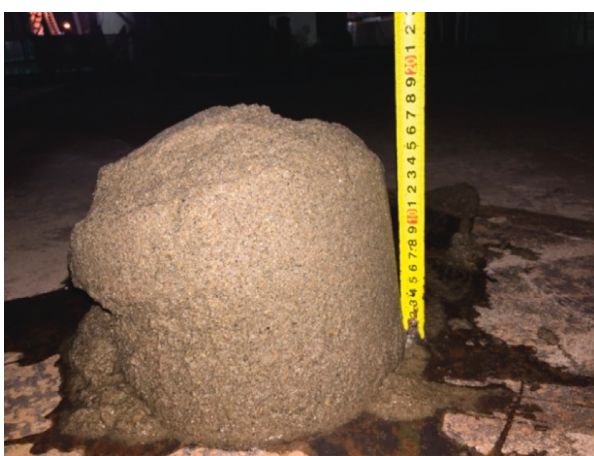

(a)

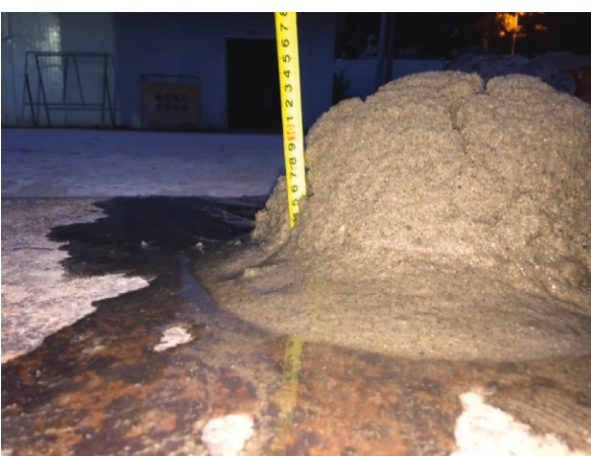

(b)

FIGURE 9: Effect of sodium bentonite-CMC mixed mud and different concentrations of polyacrylamide solution on slump improvement. (a) $3 \%$ concentration of polyacrylamide. (b) $4 \%$ concentration of polyacrylamide.

TABLE 5: Optimal mixing ratio of sodium bentonite-CMC and mud-polyacrylamide solution.

\begin{tabular}{lccc}
\hline $\begin{array}{l}\text { Sodium bentonite } \\
\text { concentration }\end{array}$ & CMC mass ratio & Polyacrylamide solution concentration & $\begin{array}{c}\text { Supersaturated sand: sodium bentonite-CMC } \\
\text { mud: polyacrylamide volume ratio }\end{array}$ \\
\hline $10 \%$ & $0.5 \%$ & $3-4 \%$ & $100: 2.5: 0.5$ \\
\hline
\end{tabular}

existing research, the effective rule of mix proportion combined with sodium bentonite-carboxymethyl cellulose mixed slurry and polyacrylamide solution on the flow ability and permeability is clarified, and the best improvement program suitable for saturated sand is determined. The field sampling tests are also conducted. The main results obtained from the study are as follows:

(1) CMC will connect the tiny particles in the soil to increase the viscosity between the particles; consequently, the improved slag will form a better waterproof sealing effect and reduce the permeability of the slag. The concentration of sodium bentonite is $11 \%, 10 \%, 9 \%$, and $8 \%$ mixed with CMC concentrations of $2.5 \%$, $5 \%$, $7.5 \%$, and $10 \%$, respectively, composing sodium bentonite-CMC mixed mud. While the sodium bentonite-CMC mixed mud is mixed with saturated sand within a certain blending range, it can meet the requirement of viscosity and permeability coefficient. Although the slump of saturated sand is also improved, it still does not meet 

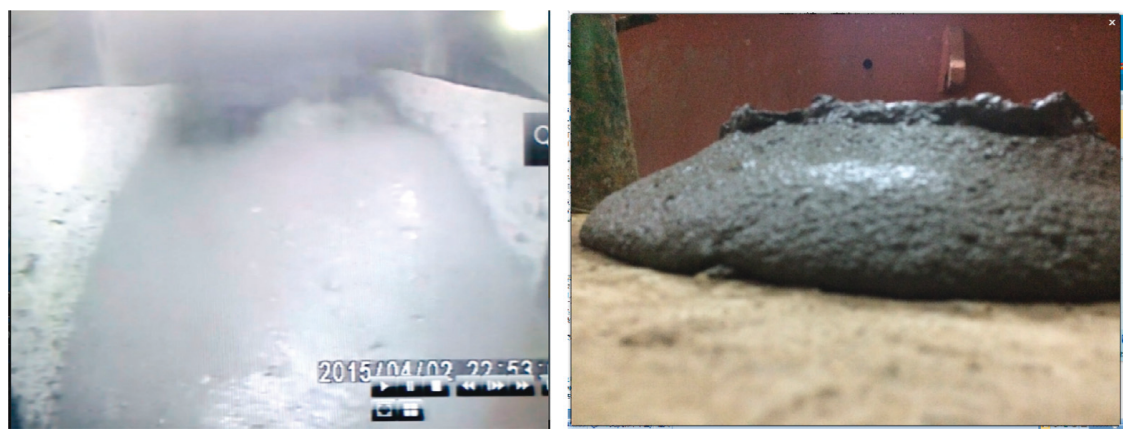

FIGURE 10: Improved morphology of the slag during the construction of EPBS.

TABLE 6: Improved slump and permeability coefficient during construction.

\begin{tabular}{lcc}
\hline Sample number & $\begin{array}{c}\text { Permeability coefficient } \\
\left(\times 10^{-6} \mathrm{~cm} / \mathrm{s}\right)\end{array}$ & Slump $(\mathrm{cm})$ \\
\hline 1 & 2.41 & 14.7 \\
2 & 1.84 & 16.3 \\
3 & 2.58 & 13.1 \\
4 & 1.75 & 14.0 \\
5 & 1.92 & 17.6 \\
6 & 2.96 & 15.2 \\
7 & 2.22 & 13.7 \\
8 & 2.25 & 15.8 \\
9 & 1.60 & 14.2 \\
\hline
\end{tabular}

the evaluation standard of $10-15 \mathrm{~cm}$, which consequently cannot meet the requirements of antispray as well.

(2) The combination of sodium bentonite-CMC mixed mud and polyacrylamide solution can provide better performance of different improved materials to meet the workability and permeability index of the sand. In the supersaturated sand stratum, the sodium bentonite-CMC mixed mud with a bentonite concentration of $10 \%$, a CMC concentration of $5 \%$, and a polyacrylamide solution with a concentration in the range of 3 to $4 \%$ were mixed with volumes of $2.5 \%$ and $0.5 \%$ of the sand. This met the needs of antispray in construction.

\section{Data Availability}

The data used to support the findings of this study are available from the corresponding author upon request.

\section{Conflicts of Interest}

The authors declare that they have no conflicts of interest in this work.

\section{Acknowledgments}

The authors appreciate the support of the National Natural Science Foundation of China (Grant no. 51578461) and the National Key Research and Development Program of China (Grant no. 2016YFC0802202).

\section{References}

[1] M. Herrenknecht, M. Thewes, and C. Budach, "The development of earth pressure shields: from the beginning to the present/entwicklung der erddruckschilde: von den anfängen bis zur gegenwart," Geomechanics and Tunnelling, vol. 4, no. 1, pp. 11-35, 2011.

[2] M. Hashimoto, S. Ryu, M. Yoshihiro, and Asada, "An empirical study on the soil management of shield facing," in Proceedings of the 29th Geological Engineering Research Presentation Paper, pp. 1965-1966, Tokyo, Japan, 1994.

[3] H. Yanagi and M. Higashi, "Flow analysis of sediment in the chamber of a large cross section soil and shield machine digger," in Proceedings of the 51st Geological Engineering Research Presentation Paper, pp. 302-303, Osaka, Japan, 1996.

[4] Sasaki, "Sediment wear simulation in the large earth pressure earth shield excavator chamber," in 51th JSSE presentation papers, pp. 304-305, 1996.

[5] G. Zheng, X. Dai, and Y. Diao, "Parameter analysis of water flow during EPBS tunnelling and an evaluation method of spewing failure based on a simplified model," Engineering Failure Analysis, vol. 58, pp. 96-112, 2015.

[6] W. Zhu, J. S. Qin, and K. L. Wei, "Research on the mechanism of the spewing in the EPB shield tunnelling," Chinese Journal of Geotechnical Engineering, vol. 26, no. 5, pp. 589-593, 2004.

[7] J. S. Qin and W. Zhu, "Study on flowing through screw conveyor during excavation with EPB shield," Rock and Soil Mechanics, vol. 25, no. 10, pp. 1632-1636, 2004.

[8] Y. W. Han, X. C. Zhong, W. Zhu, and X. F. Yu, "Soil conditioning influence on face stability of shield tunnel," Rock and Soil Mechanics, vol. 28, no. 1, pp. 516-520, 2007.

[9] W. B. Zhu, S. J. Ju, and M. Zhang, "On the engineering poser and countermeasures of driving and crossing the pearl river with two used TBMs in Guangzhou metro line of no. 2," China Civil Engineering Journal, vol. 37, no. 1, pp. 56-60, 2004.

[10] Q. X. Yan, D. J. Zheng, and C. He, "Problems and countermeasures of metro shield construction in water-soaked sand and cobble stratum," Chinese Journal of Underground Space and Engineering, vol. 11, no. 3, pp. 713-719, 2015.

[11] D. Peila, "Soil conditioning for EPB shield tunnelling," KSCE Journal of Civil Engineering, vol. 18, no. 3, pp. 831-836, 2014.

[12] C. Oggeri, T. M. Fenoglio, and R. Vinai, "Tunnel spoil classification and applicability of lime addition in weak formations for muck reuse," Tunnelling and Underground Space Technology, vol. 44, no. 3, pp. 97-107, 2014.

[13] C. Oggeri, "Tunnelling muck classification: definition and application," in Proceedings of the World Tunnel Congress 2017, Bergen, Norway, June 2017. 
[14] C. Oggeri, T. M. Fenoglio, and R. Vinai, "Muck classification: raw material or waste in tunnelling operation," Revista Minelor, vol. 20, no. 4, pp. 16-25, 2014.

[15] S. Quebaud, M. Sibai, and J.-P. Henry, "Use of chemical foam for improvements in drilling by earth-pressure balanced shields in granular soils," Tunnelling and Underground Space Technology, vol. 13, no. 2, pp. 173-180, 1998.

[16] P. Sotiris, "Properties of foam/sand mixtures for tunnelling applications," A thesis submitted for the degree of Master of Science to the Department of Engineering Science, University of Oxford, Oxford, UK, 2001.

[17] B. Xavier, "Soil conditioning for clay soil," Tunnels and Tunnelling International, vol. 4, pp. 29-32, 2003.

[18] D. Peila, C. Oggeri, and R. Vinai, "Screw conveyor device for laboratory tests on conditioned soil for EPB tunneling operations," Journal of Geotechnical and Geoenvironmental Engineering, vol. 133, no. 12, pp. 1622-1625, 2007.

[19] D. Peila, A. Picchio, and A. Chieregato, "Earth pressure balance tunnelling in rock masses: laboratory feasibility study of the conditioning process," Tunnelling and Underground Space Technology, vol. 35, no. 35, pp. 55-66, 2013.

[20] A. G. Ehsan, R. Jamal, and T. Kaveh, "Experimental study of the effect of conditioning on abrasive wear and torque requirement of full face tunneling machines," Tunnelling and Underground Space Technology, no. 3, pp. 127-136, 2014.

[21] S. H. He, S. C. Zhang, and C. H. Li, "Blowout control during EPB shield tunnelling in sandy pebble stratum with high groundwater pressure," Chinese Journal of Geotechnical Engineering, vol. 39, no. 09, pp. 1583-1590, 2017.

[22] D. Martinelli, D. Peila, and E. Campa, "Feasibility study of tar sands conditioning for earth pressure balance tunnelling," Journal of Rock Mechanics and Geotechnical Engineering, vol. 7, no. 6, pp. 684-690, 2015.

[23] J. Y. Peron and P. Marcheselli, "Construction of the Passante Ferroviario link in Milan, Italy, lots 3P, 5P and 6P: excavation by large earth pressure balanced shield with chemical foam injection," in Tunnelling 94, Springer, Berlin, Germany, 1994.

[24] S. Jancsecz, R. Krause, and L. Langmaack, "Advantages of soil conditioning in shield tunneling: experiences of LRTS Izmir," in Proceedings of the International Congress on Challenges for the 21st Century, pp. 865-875, Balkema, Oslo, Norway, June 1999.

[25] G. E. Williamson, M. T. Traylor, and M. Higuchi, "Soil conditioning for EPB shield tunneling on the South Bay Ocean Outfall," in Proceedings of the Rapid Excavation and Tunneling Conference 1999, D. Hilton and K. Samuelson, Eds., pp. 897-925, SME, Orlando, FL, USA, June 1999.

[26] M. Peña, "Soil conditioning for sands," in Tunnels and Tunnelling International, vol. 35, no. 7, pp. 40-44, 2003.

[27] S. J. Boone, E. Artigiani, J. N. Shirlaw, R. Ginanneschi, T. Leinala, and N. Kochmanova, "Use of ground conditioning agents for earth pressure balance machine tunneling," in Proceedings of the AFTES International Congress, Chamberry, pp. 313-319, Chambéry, France, October 2005.

[28] M. Thewes and C. Budach, "Soil conditioning with foam during EPB tunnelling konditionierung von lockergesteinen bei erddruckschilden," Geomechanics and Tunnelling, vol. 3, no. 3, pp. 256-267, 2010.

[29] D. Peila, A. Picchio, D. Martinelli, and E. D. Negro, "Laboratory tests on soil conditioning of clayey soil," ActaGeotechnica, vol. 9, pp. 1-14, 2015.

[30] Y. S. Liu and Q. Z. Bai, "Study on bentonite enhanced soil as landfill materials," Journal of Basic Science and Engineering, vol. 2, pp. 143-149, 2002.
[31] R. Vinai, C. Oggeri, and D. Peila, "Soil conditioning of sand for EPB applications: a laboratory research," Tunnelling and Underground Space Technology, vol. 23, no. 3, pp. 308-317, 2008.

[32] G. Anagnostou and K. Kovári, "Face stability conditions with earth-pressure-balanced shields," Tunnelling and Underground Space Technology, vol. 11, no. 2, pp. 165-173, 1996.

[33] K. Yang, "Analysis and application of water-swelling law of montmorillonite," Sichuan Building Science, vol. 34, no. 5, pp. 152-154, 2008.

[34] X. Xia, The Research on Soil Conditioning of Tunneling in High Hydraulic Pressure and High Permeability Sandy Stratum, Southwest Jiaotong University, Chengdu, China, 2016.

[35] Z. L. Wang, Research on Improvement Technology of Slag Soil in Underwater Tunnel of Nanchang Subway, Nanchang Institute of Technology, Nanchang, China, 2015.

[36] M. S. Wang, "Muck conditioning technology in complex ground for tunnel by shield tunneling method," Chinese Journal of Underground Space and Engineering, vol. 3, no. 2, pp. 1445-1463, 2007.

[37] F. J. Guo, Z. W. Zhao, and J. Zhang, "Experimental study of ground conditioning of pure sand stratum by using polymer," Tunnel Construction, vol. 37, no. 1, pp. 53-58, 2017.

[38] Y. Y. Wang, Research of a New Soil Improvement for Sand and Gravel EPS Shield, China University of Geosciences, Wuhan, China, 2010. 


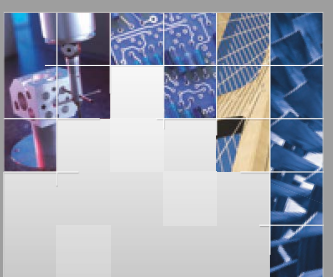

\section{Enfincering}
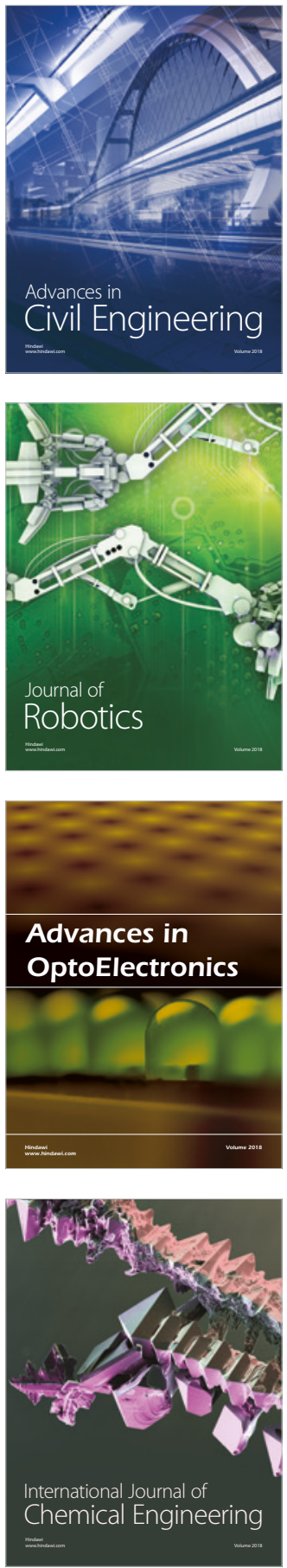

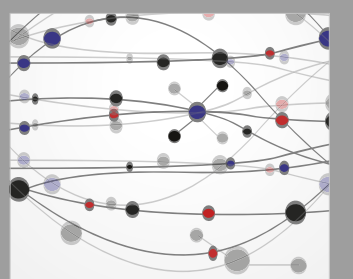

\section{Rotating \\ Machinery}

The Scientific World Journal

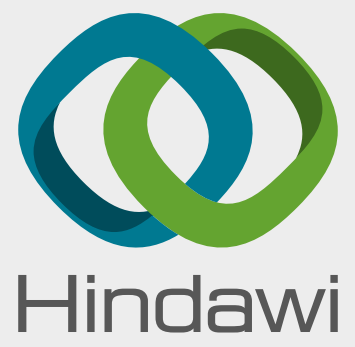

Submit your manuscripts at

www.hindawi.com
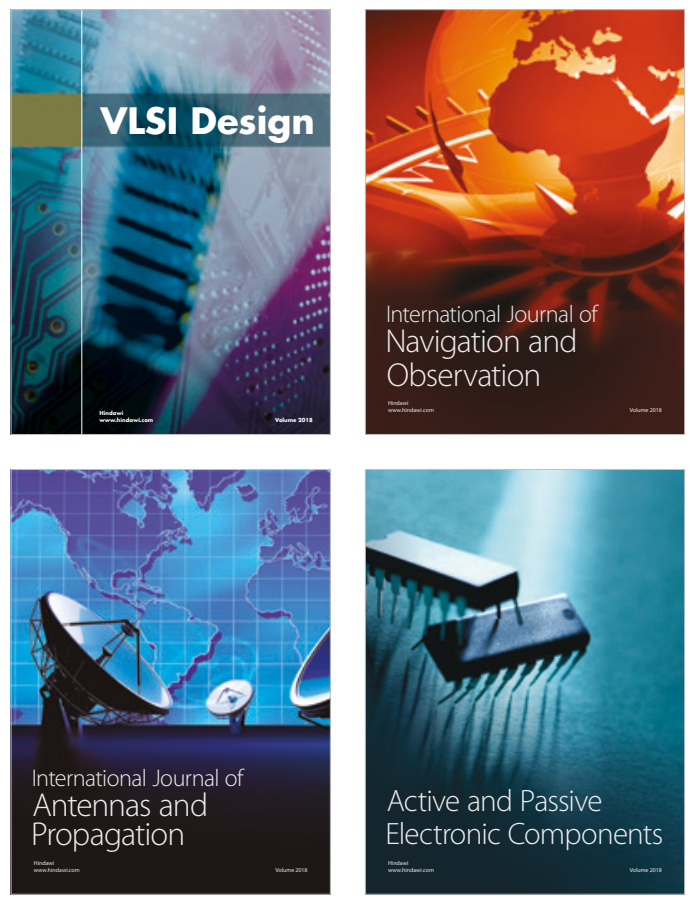
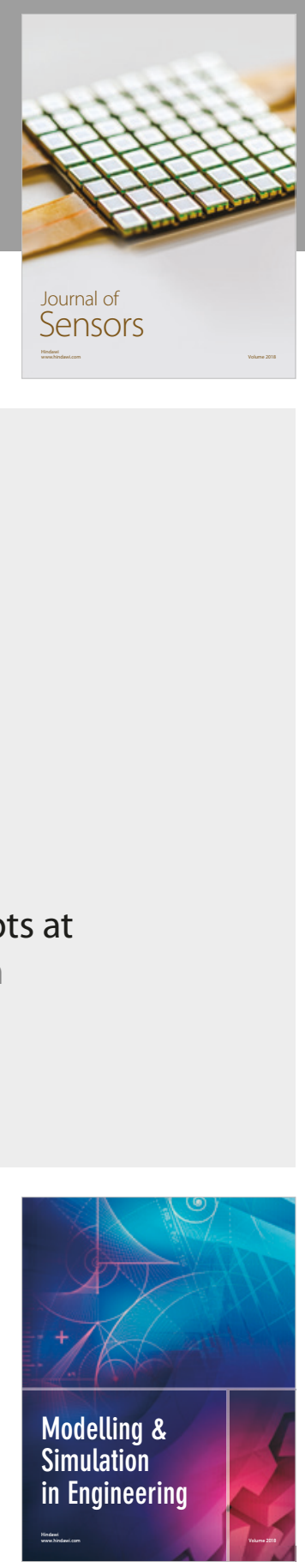

\section{Advances \\ Multimedia}
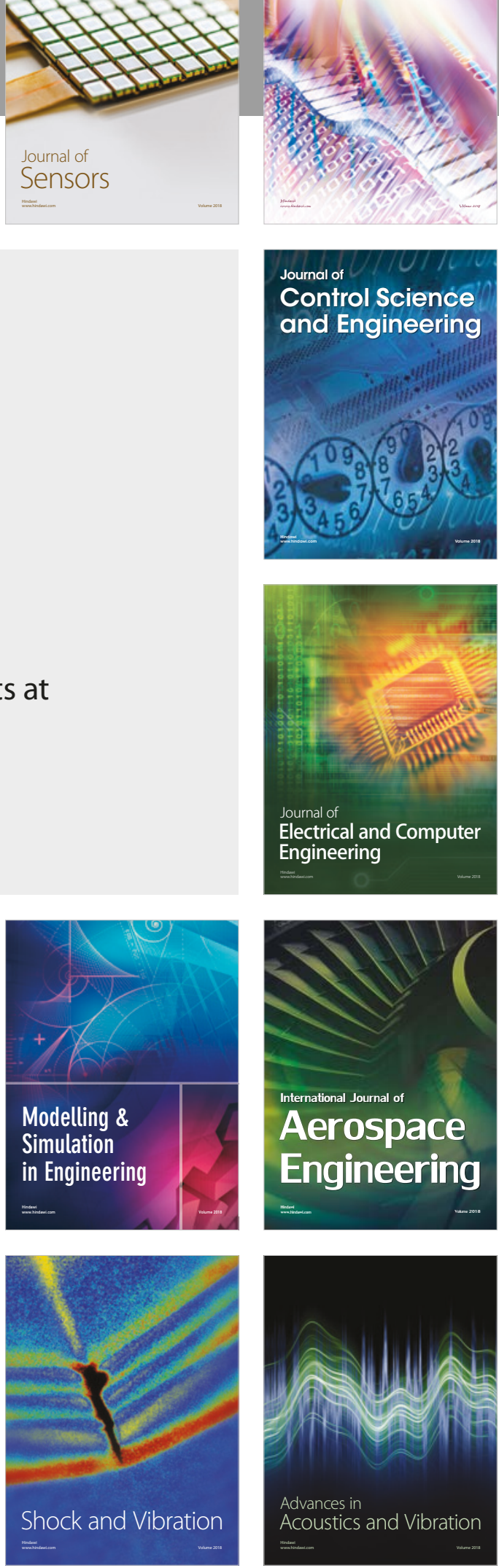